RITS-PP-002

\title{
de Vaucouleurs-Ikeuchi Diagram and Commutation Relations among Phase Space Coordinates
}

\author{
Takeshi FUKUYAMA ${ }^{1}$ and Tatsuru KIKUCHI ${ }^{2}$ \\ Department of Physics, Ritsumeikan University, \\ Kusatsu, Shiga, 525-8577, Japan
}

\begin{abstract}
We consider the relations between de Vaucouleurs-Ikeuchi diagram and generalized commutation relations among the coordinates and momenta. All physical objects in the Universe ranging from elementary particles to super cluster of galaxies are confined within the Triangle of the de VaucouleursIkeuchi diagram on the matter density versus scale length plane. These three boundaries are characterized by the quantum uncertainty principle, gravitational event horizon, and cosmological constant. These are specified by the non-zero commutation relations $\left[x_{\mu}, p_{\nu}\right],\left[x_{\mu}, x_{\nu}\right]$ (strictly $\left[x_{i}, t\right]$ ) and $\left[p_{\mu}, p_{\nu}\right]$, respectively. The canonical commutation relation $\left[x_{i}, p_{j}\right]$ are slightly modified, which preserves the self consistency as a whole.
\end{abstract}

\section{Introduction}

All physical entities ranging from microscopic quark-leptons to macroscopic super cluster of galaxies are confined within the Triangle in the de VaucouleursIkeuchi diagram [1] on log-log scale of matter density versus cosmological length (Fig.1).

The line A corresponds to the 'quantum limiting line' beyond (toward lower left) which all the structures are perturbed by quantum fluctuations and cannot exist as observable bodies. The limiting line is given as follows: the Compton

\footnotetext{
${ }^{1}$ E-mail: fukuyama@se.ritsumei.ac.jp

2E-mail: rp009979@se.ritsumei.ac.jp
} 


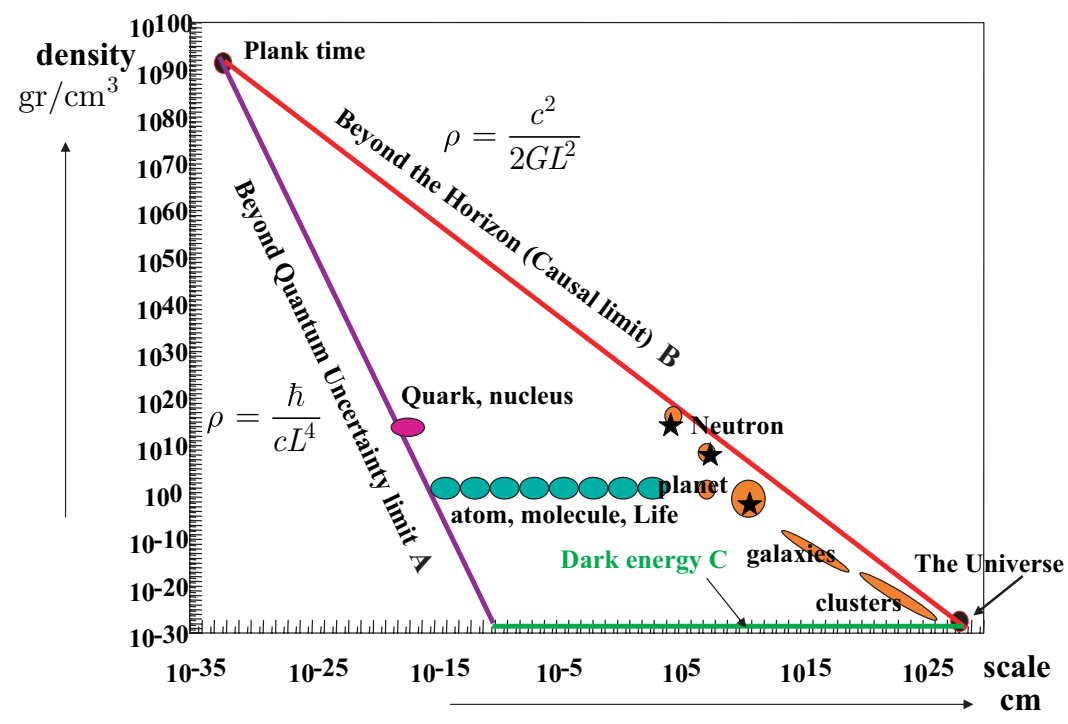

Figure 1: The de Vaucouleurs-Ikeuchi diagram and the Triangle

length associated with a particle of mass $M$

$$
\lambda=\frac{h}{M c}
$$

which is the smallest de Broglie wave length associated with matter $\lambda=\frac{h}{M v}$. The corresponding mass density is given by

$$
\rho_{\text {quantum }}=\frac{M}{(4 \pi / 3) \lambda^{3}}=\frac{h}{(4 \pi / 3) c L^{4}} .
$$

The line B corresponds to the 'causal limiting line' beyond (toward upper right) which all the structures collapse gravitationally and form black holes which have no individuality. The limiting line is given by considering gravitational (Schwarzshild) radius

$$
L=\frac{2 G M}{c^{2}}
$$


The limiting mass density is obtained by

$$
\rho_{\text {causal }}=\frac{M}{(4 \pi / 3) L^{3}}=\frac{c^{2}}{(4 \pi / 3) G L^{2}},
$$

and therefore inversely proportional to the length squared.

The horizontal line $\mathrm{C}$ corresponds to the cosmological constant $\left(\rho_{\text {const }}\right)$ [2] below which no mass density is attainable.

All observable constituents in the Universe are within the triangle enclosed by these three edges. The first uncertainty principles are the realization of

$$
\left[x_{\mu}, p_{\nu}\right]=i \hbar \eta_{\mu \nu}
$$

and leads us to the quantum uncertainty principle

$$
\Delta x \Delta p \geq \hbar, \quad \Delta t \Delta E \geq \hbar .
$$

(Here and hereafter we use natural units except for the case to emphasize $c$ or $\hbar$.) As we will show, these relations are slightly modified in the total framework. Before discussing the line B we proceed to study the line $\mathrm{C}\left(\rho_{\text {const }}\right)$, which is the dark energy or cosmological constant.

$$
\rho_{\text {const }}=10^{-122} M_{\mathrm{Pl}}^{4} .
$$

The cosmological constant is formulated in de Sitter invariant gravity [3]. The Einstein-de Sitter gravity Lagrangian

$$
\mathcal{L}=\partial_{\mu} K^{\mu}-\frac{1}{16 \pi G_{N}} \sqrt{-g}\left(R+\frac{6}{\ell^{2}}\right)
$$

which is invariant under the de Sitter gauge group

$$
\eta_{A B} X^{A} X^{B}=\ell^{2}(A, B=0, ., 4) .
$$

Hence the cosmological constant is given by

$$
\rho_{\text {const }}=\frac{3}{8 \pi \ell^{2} G_{N}} .
$$

The inflationary coordinates $\left(t, x^{i}\right)$ are defined by

$$
\begin{aligned}
& X^{0}=\ell \sinh (t / \ell)+\frac{\left(x^{i}\right)^{2}}{2 \ell} e^{t / \ell}, \\
& X^{i}=x^{i} e^{t / \ell}, \\
& X^{4}=\ell \cosh (t / \ell)-\frac{\left(x^{i}\right)^{2}}{2 \ell} e^{t / \ell} .
\end{aligned}
$$


This leads to the metric in the inflationary era,

$$
d s^{2}=-d t^{2}+e^{2 t / \ell}\left(d x^{i}\right)^{2} .
$$

Hence the size of the Universe grows as $\sim e^{2 t / \ell}$. On the other hand, the static metric is given by

$$
d s^{2}=-\left(1-\frac{r^{2}}{\ell^{2}}\right) d t^{2}+\frac{d r^{2}}{\left(1-\frac{r^{2}}{\ell^{2}}\right)}+r^{2} d \Omega_{2}^{2} .
$$

Note that the line element becomes singular for $r=\ell$, which is a cosmological event horizon to which we can associate an entropy

$$
S=\frac{2 \pi \ell^{2}}{G_{N}} .
$$

The Penrose diagram is shown in Fig. 2. The peculiar feature of de Sitter space

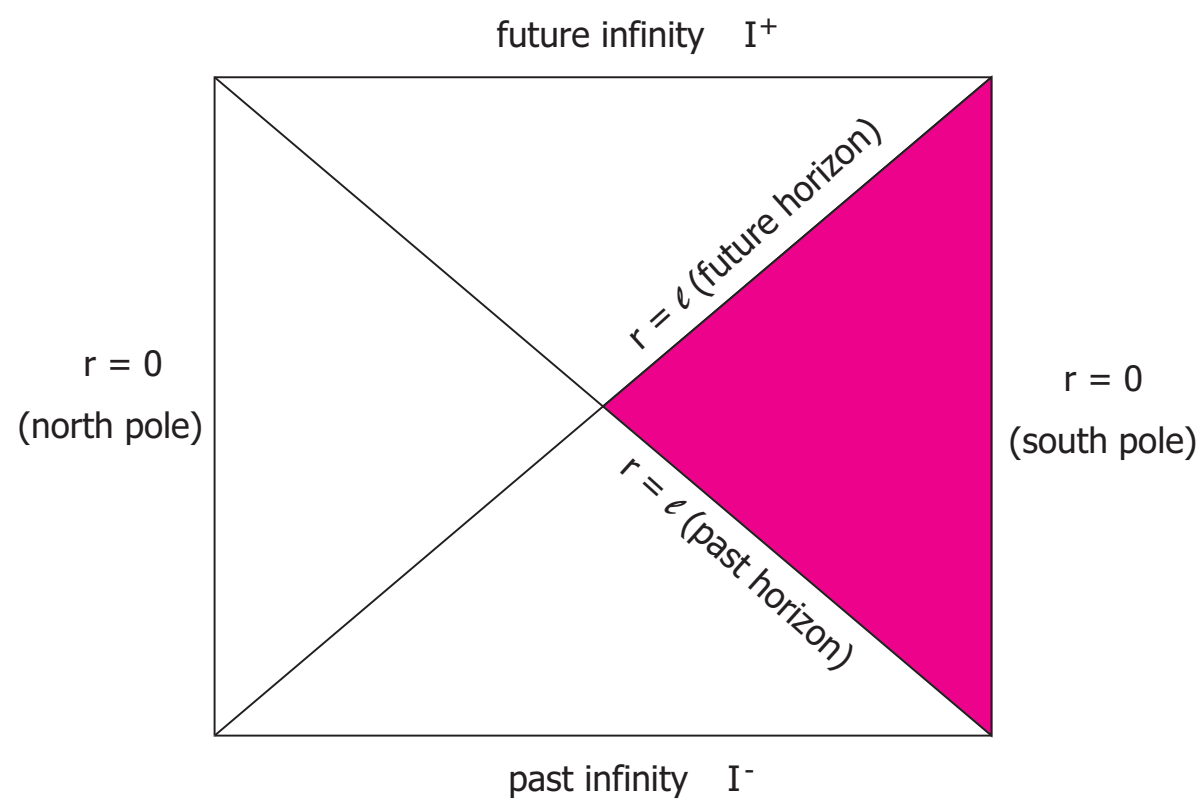

Figure 2: The Penrose diagram for de Sitter space [4].

is that no single observer can acccess the entire space-time. In the diagram, an observer sitting on the south pole will never be able to observe anything past the 
diagonal line stretching from the north pole at $\mathcal{I}^{-}$to the south pole at $\mathcal{I}^{+}$, and the shaded region is called as "causal diamond". For more details, see, e.g. [5].

The generators of de Sitter group transformation satisfy the commutation relation

$$
\left[M_{A B}, M_{C D}\right]=i \hbar\left(\eta_{A C} M_{B D}+\eta_{B D} M_{A C}-\eta_{A D} M_{B C}-\eta_{B C} M_{A B}\right)(A, . ., D=0, . ., 4)
$$

Momenta $p_{\mu}(\mu=0, . ., 3)$ are defined by

$$
p_{\mu}=M_{\mu 4} / \ell
$$

and they are not commutative to each others,

$$
\left[p_{\mu}, p_{\nu}\right]=i \hbar \ell^{-2} M_{\mu \nu} .
$$

It should be remarked that (15) reduces to the Poincaré algebra in the WignerInönü contraction $\ell \rightarrow \infty$.

Thus the line $\mathrm{A}$ and the line $\mathrm{C}$ are characterised by $\left[x_{\mu}, p_{\nu}\right] \neq 0$ and $\left[p_{\mu}, p_{\nu}\right] \neq$ 0 , respectively. So we are attracted to think that the remaining line B reflects new physics related with $\left[x_{\mu}, x_{\nu}\right] \neq 0$. We will show in the following that this is indeed the case.

At first we may postulate

$$
\left[x_{i}, x_{j}\right]=i \theta_{i j}(i, j=1,2,3)
$$

as in the non-commutative geometry [6]. However, we can not associate this with event horizon, though its possibility can not be refused. Instead, we adopt the Yoneya's relation in string theory [7].

$$
\left[x_{i}, t\right]=\ell_{s}^{2}=\alpha^{\prime}
$$

where $\ell_{s}$ is the string length. This is necessary condition to be free from ultraviolet divergence and can be interpreted also by considering the scattering of two D-particles with impact parameter of order $\Delta X \geq \ell_{s}$ and obtain

$$
\Delta X \Delta T \geq \ell_{s}^{2}
$$

As was shown in [8] (19) in string theory is free from the acausal mode unlike the same case of the simple non-commutative geometry (18). The implications of the stringy space-time uncertainty (20) on the spectrum of cosmological fluctuations was studied in [9]. 
Considering the event horizon of the size of $\ell_{s}$, we can obtain the bonus to reproduce the Bekenstein-Hawking entropy $S_{\mathrm{BH}}$ relation [10] from microscopic sum of quantum states,

$$
\log d(E) \approx G_{N}^{\frac{1}{D-3}} E^{\frac{D-2}{D-3}} \approx S_{\mathrm{BH}} \equiv \frac{\text { Area of } \mathrm{BH}}{4 G_{N}} .
$$

Here $d(E)$ is a quantum degeneracy,

$$
d(E)=\exp \left(2 \pi \sqrt{(D-2) / 6} \ell_{s} E\right),
$$

and $D$ is the space-time dimension.

Now using the recent advances in string theory of AdS/CFT correspondence we can know about a connection among two scales $\ell$ and $\ell_{s}$. That is, there is a duality between the $\mathrm{AdS}_{5} \times \mathrm{S}_{5}$ compactification of the type IIB string theory and the superconformal Yang-Mills theory of the $\mathrm{SU}(N)$ gauge group [11]. Thus the de Sitter radius $\ell$ is given in terms of the string scale $\ell_{s}$ and the gauge index $N$ as

$$
\ell=\left(4 \pi g_{s} N\right)^{1 / 4} \ell_{s}
$$

where $g_{s}=\sqrt{8 \pi G_{N} / \alpha^{\prime}}$ is the string coupling constant. For the more detailed argument of the de Sitter entropy, see, e.g. [12].

So far we have considered the extended canonical commutation relations. Here we argue on the consistency of those relations. We assume that the Jacobi identity still holds in this extended commutation relations. We consider

$$
\left[\left[p_{i}, p_{j}\right], x_{k}\right]+\left[\left[x_{k}, p_{i}\right], p_{j}\right]+\left[\left[p_{j}, x_{k}\right], p_{i}\right]=0 .
$$

It goes from (17) that the first term is

$$
\left[\left[p_{i}, p_{j}\right], x_{k}\right]=i \hbar \ell^{-2}\left[M_{i j}, x_{k}\right]=-\hbar \ell^{-2}\left(\delta_{i k} x_{j}-\delta_{j k} x_{i}\right)
$$

Hence to preserve the Jacobi's identity we must extend the canonical commutation relation as

$$
\left[x_{i}, p_{j}\right]=i \hbar \delta_{i j}\left(1+\beta x^{2}\right) .
$$

Here $\beta$ is a tiny parameter to be constrained from (25) as

$$
\beta=-\frac{1}{2 \ell^{2}}
$$


The different extension of $\left[x_{i}, p_{j}\right]$ from ours was considered in [13],

$$
\left[x_{i}, p_{j}\right]=i \hbar \delta_{i j}\left(1+\beta^{\prime} p^{2}\right)
$$

which leads to the minimal length $\Delta x_{\min } \geq \hbar \sqrt{\beta^{\prime}} \approx \ell_{s}$. So different tiny corrections to canonical commutation relations induces enormously different length scale $\ell$ and $\ell_{s}$. If we consider another type of the Jacobi identity

$$
\left[\left[x_{i}, p_{j}\right], x_{k}\right]+\left[\left[x_{k}, x_{i}\right], p_{j}\right]+\left[\left[p_{j}, x_{k}\right], x_{i}\right]=0 .
$$

(29) is reduced from (26) to

$$
\beta \delta_{i j}\left[x^{2}, x_{k}\right]+\beta \delta_{j k}\left[x^{2}, x_{i}\right]+\left[\left[x_{k}, x_{i}\right], p_{j}\right]=0 .
$$

This does not allow the extension of

$$
\left[x_{i}, x_{j}\right]=0 .
$$

It should be remarked that the above arguments do not restrict $[t, x]$ commutation relation. These commutation relations allows the representation

$$
\hat{x}_{i}=x_{i}, \quad \hat{p}_{j}=-i \hbar\left(1+\beta x^{2}\right) \frac{\partial}{\partial x_{i}}
$$

unlike (18).

The commutation relation $\left[p_{i}, p_{j}\right]$ leads to (27) again. If we consider [14]

$$
\int\left|\alpha x \psi+\left(1+\beta x^{2}\right) \psi\right|^{2} d x \geq 0
$$

for arbitrary real $\alpha$, we obtain the generalized uncertainty relation

$$
\Delta p \geq \frac{\hbar}{2}\left(\frac{1}{\Delta x}+3 \beta \Delta x\right),
$$

which implies the minimum momentum

$$
\Delta p_{\min } \geq \frac{\hbar}{\ell} .
$$

Using the generalized Poisson bracket $\{A, B\}_{\text {P.B. }}$ obtained from the correspondence,

$$
\frac{[A, B]}{i \hbar}=\{A, B\}_{\text {P.B. }}
$$


we can show that the Liouville theorem holds,

$$
\frac{d^{D-1} x^{\prime} d^{D-1} p^{\prime}}{\left(1+\beta x^{2}\right)^{D-1}}=\frac{d^{D-1} x d^{D-1} p}{\left(1+\beta x^{2}\right)^{D-1}}
$$

thanks to (27). Obviously this does not serve as the suppression of cosmological constant like [15]. However the correct magnitude of cosmological constant is guaranteed as the lowest energy of de Sitter invariant vacuum [3].

Unfortunately we can not find the bridge between the cosmological constant and the string length scale. One of the reasons is that the ordinary commutation relations are concerned the spatial coordinates of phase space. On the other hand, the string length scale appeared in the commutation relation between space and time coordinates.

Consequently we have discussed the Triangle in the de Vaucouleurs-Ikeuchi diagrams. The three boundaries characterise quantum mechanics, black hole, and cosmological constant, which are described by the non-zero commutation relations, $\left[x_{i}, p_{j}\right],\left[t, x_{i}\right]$, and $\left[p_{i}, p_{j}\right]$, respectively. The first canonical commutation relation is modified to $\left[x_{i}, p_{j}\right]=i \hbar \delta_{i j}\left(1+\beta x^{2}\right)$, which preserves the self consistency as a whole. This is the peculiar property of de Vaucouleurs-Ikeuchi diagram (log density-log length diagram).

Acknowledgments

T.F. is grateful to M. Morikawa for useful comments and helps. He also thanks C.Q. Geng at NCTS for discussions and High Energy group at NCTS and Theory Division at KEK for hospitality. This work is supported by the Grant-in-Aid for Scientific Research from the Ministry of Education, Science and Culture of Japan (\#16540269). The work of T.K. is supported by the Research Fellowship of the Japan Society for the Promotion of Science (\#7336).

\section{References}

[1] G. de Vaucouleurs, Science 1671203 (1970); S. Ikeuchi, "Kansokuteki Utyuuron (Introduction to Observational Cosmology)" in Japanese (University of Tokyo Press, 1997).

[2] C. L. Bennett et al., "First Year Wilkinson Microwave Anisotropy Probe (WMAP) Observations: Preliminary Maps and Basic Results," Astrophys. J. Suppl. 148, 1 (2003) [arXiv:astro-ph/0302207]; D. N. Spergel et 
al. [WMAP Collaboration], "First Year Wilkinson Microwave Anisotropy Probe (WMAP) Observations: Determination of Cosmological Parameters," Astrophys. J. Suppl. 148, 175 (2003) [arXiv:astro-ph/0302209].

[3] S. W. MacDowell and F. Mansouri, "Unified Geometric Theory Of Gravity And Supergravity," Phys. Rev. Lett. 38, 739 (1977) [Erratum-ibid. 38, 1376 (1977)]; K. S. Stelle and P. C. West, "Spontaneously Broken De Sitter Symmetry And The Gravitational Holonomy Group," Phys. Rev. D 21, 1466 (1980); T. Fukuyama, "De Sitter Invariant Gravity Coupled With Matters And Its Cosmological Consequences," Annals Phys. 157, 321 (1984).

[4] S.W. Hawking and G.F.R. Ellis, The Large Scale Structure of Space-Time, (Cambridge University Press, 1973).

[5] M. Spradlin, A. Strominger and A. Volovich, "Les Houches lectures on de Sitter space," arXiv:hep-th/0110007].

[6] There are many books on this subject. Here we simply cite a few review references;

N.A. Nekrasov, "Trieste Lectures on Solitons in Noncommutative Gauge Theories", [arXive:hep-th/0011095]; J.A. Harvey, "Komaba Lectures on Noncommutative Solitons and D-Branes", [arXive:hep-th/0102076].

[7] T. Yoneya, "Duality and Indeterminacy Principle in String Theory" in "Wandering in the Fields", eds. K. Kawarabayashi and A. Ukawa (World Scientific, 1987), p. 419; "String Theory and Quantum Gravity” in "Quantum String Theory”, eds. N. Kawamoto and T. Kugo (Springer, 1988), p. 23.

[8] N. Seiberg, L. Susskind and N. Toumbas, "Space/time non-commutativity and causality," JHEP 0006, 044 (2000) [arXiv:hep-th/0005015].

[9] R. Brandenberger and P. M. Ho, "Noncommutative spacetime, stringy spacetime uncertainty principle, and density fluctuations," Phys. Rev. D 66, 023517 (2002) [AAPPS Bull. 12N1, 10 (2002)] [arXiv:hep-th/0203119].

[10] M. Li and T. Yoneya, "Short-distance space-time structure and black holes in string theory: A short review of the present status," [arXiv:hep-th/9806240]. 
[11] J. M. Maldacena, "The large N limit of superconformal field theories and supergravity," Adv. Theor. Math. Phys. 2, 231 (1998) [Int. J. Theor. Phys. 38, 1113 (1999)]; For a review of AdS/CFT correspondence, see, O. Aharony, S. S. Gubser, J. M. Maldacena, H. Ooguri and Y. Oz, "Large N field theories, string theory and gravity," Phys. Rept. 323, 183 (2000) [arXiv:hep-th/9905111].

[12] V. Balasubramanian, P. Horava and D. Minic, "Deconstructing de Sitter," JHEP 0105, 043 (2001) [arXiv:hep-th/0103171].

[13] A. Kempf, G. Mangano and R. B. Mann, "Hilbert space representation of the minimal length uncertainty relation," Phys. Rev. D 52, 1108 (1995) [arXiv:hep-th/9412167].

[14] L.D. Landau and E.M. Lifshitz, Quantum Mechanics (Pergamon Press, 1964).

[15] L. N. Chang, D. Minic, N. Okamura and T. Takeuchi, "The effect of the minimal length uncertainty relation on the density of states and the cosmological constant problem," Phys. Rev. D 65, 125028 (2002) [arXiv:hep-th/0201017]. 